\title{
Regional analysis of multivariate compound flooding potential: sensitivity analysis and spatial patterns
}

Paula Camus ${ }^{1}$, Ivan D. Haigh ${ }^{1}$, Ahmed Nasr ${ }^{2}$, Thomas Wahl ${ }^{2}$, Stephen E. Darby ${ }^{3}$, Robert J. Nicholls ${ }^{4}$

${ }^{1}$ School of Ocean and Earth Science, National Oceanography Centre Southampton, University of Southampton, Waterfront

5 Campus, European Way, Southampton, SO14 3ZH, UK.

${ }^{2}$ Civil, Environmental, and Construction Engineering \& National Center for Integrated Coastal Research, University of Central Florida, 12800 Pegasus Drive, Suite 211, Orlando, FL 32816-2450, USA.

${ }^{3}$ School of Geography and Environmental Sciences, University of Southampton, Avenue Campus, Highfield Road, Southampton, SO17 1BJ, United Kingdom.

$10{ }^{4}$ Tyndall Centre for Climate Change Research, School of Environmental Sciences, University of East Anglia, Norwich, NR4 7TJ, United Kingdom.

Correspondence to: Paula Camus (P.Camus-Brana@soton.ac.uk)

\section{SUPLEMENTARY MATERIAL}

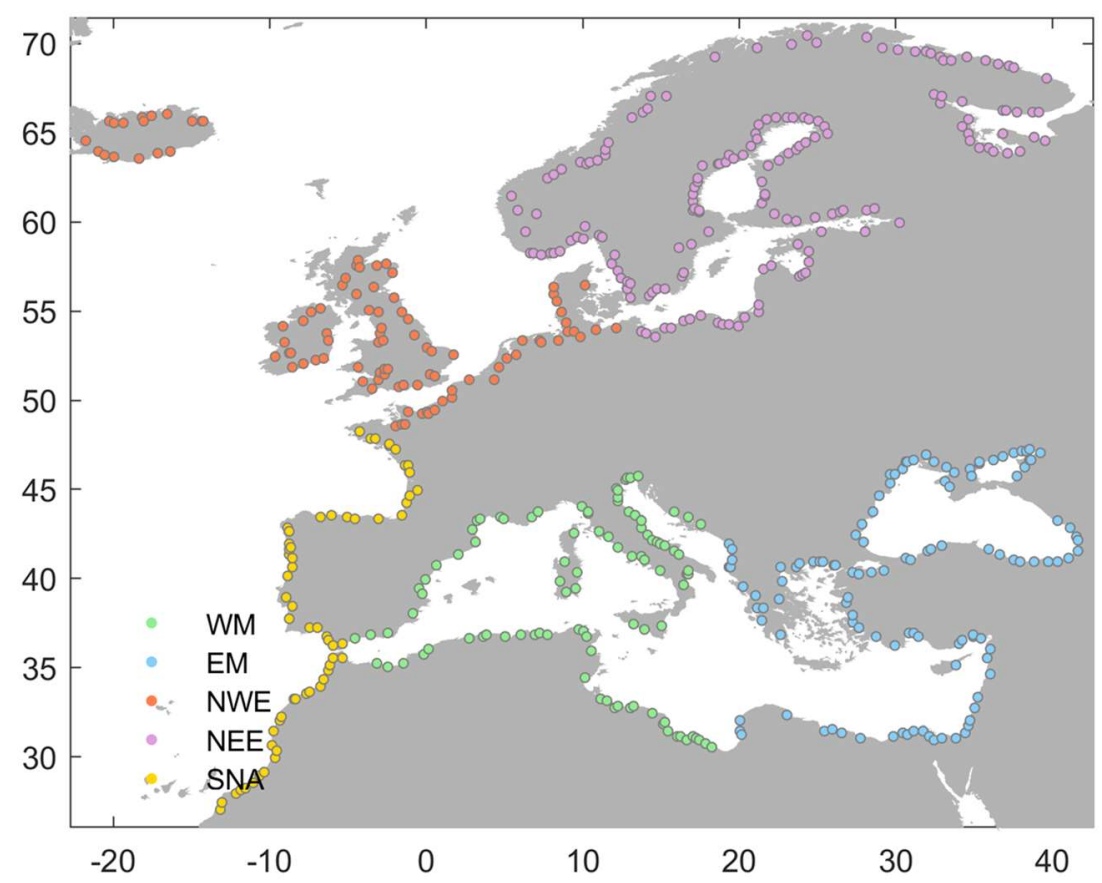

Figure S1: The study domain divided into five regions: NW (Northwestern Europe); NE (Northeastern Europe); SNA (Southern North Atlantic Ocean); WM (Western Mediterranean Sea) and EM (Eastern Mediterranean Region). 

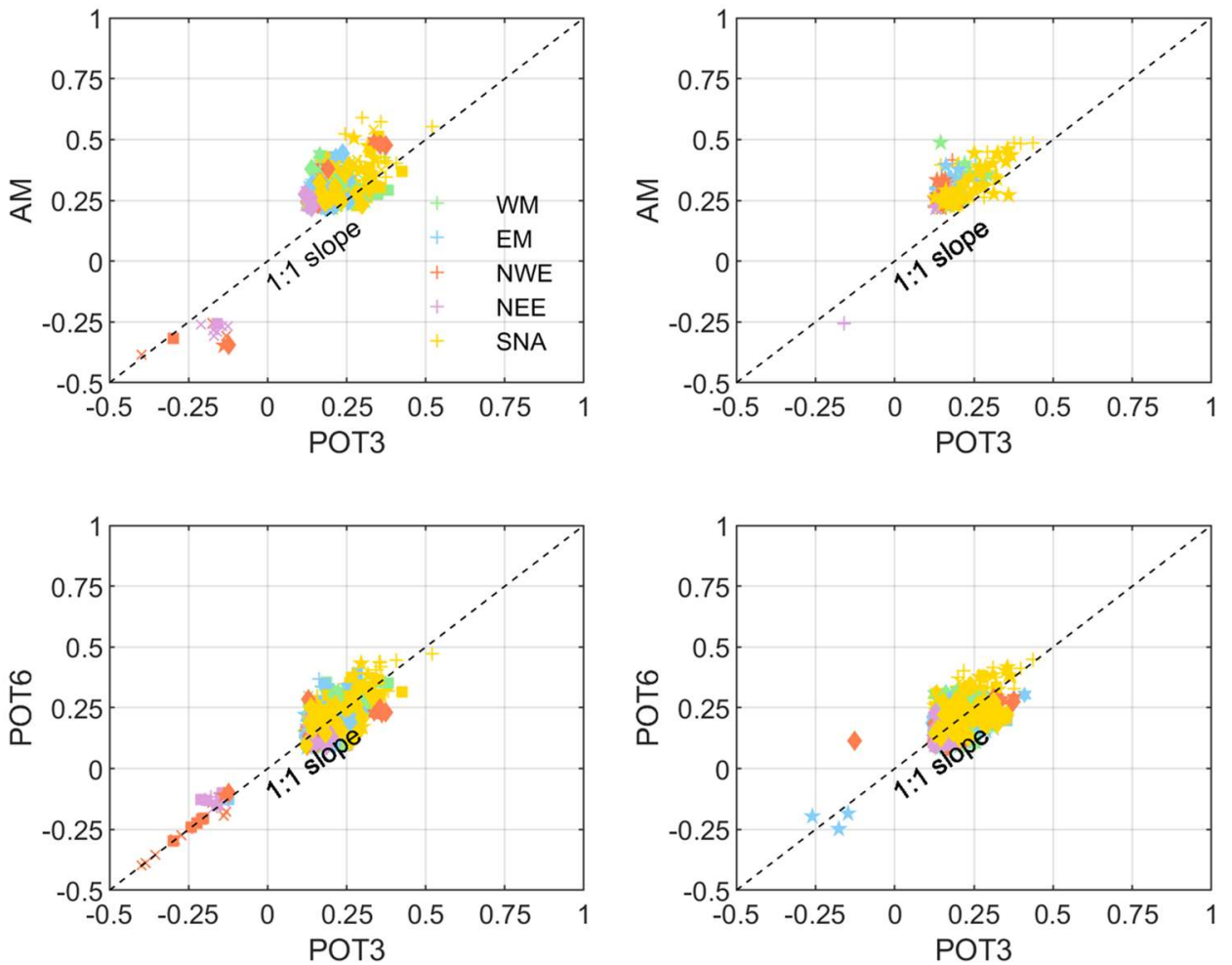

Figure S2: Scatter plots of the Kendall's correlation between Q-S (+), Q-W (x), Q-SW (•), P-S (•), P-W (*), P-SW (•), using POT3 vs AM (upper panels) and POT3 vs POT6 (lower panels). The left panels indicate when $Q$ or $P$ is the dominant variable, compared to the use of $\mathrm{S}, \mathrm{W}$ or $\mathrm{SW}$ as the dominant variable on the right panels. 

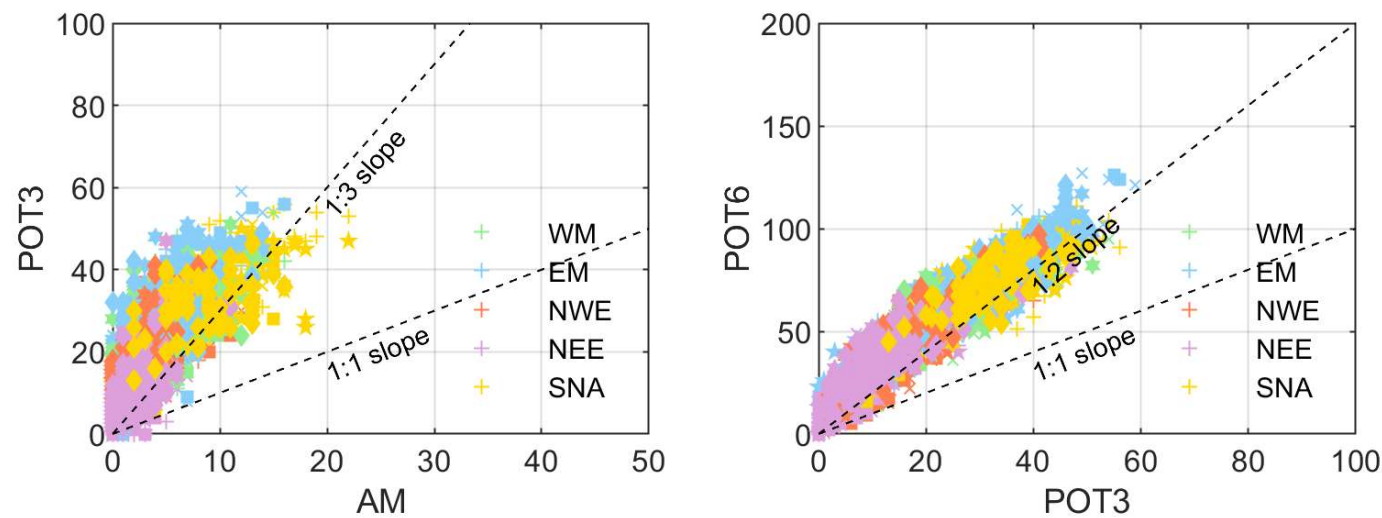

Figure S3: Scatter plots of the number of co-occurrence events between all possible combinations of pairs: Q-S (+), Q-W (x), Q-SW (•), P-S (•), P-W (*), P-SW (•), using AM vs POT3 (left panel) and POT3 vs POT6 (right panel).
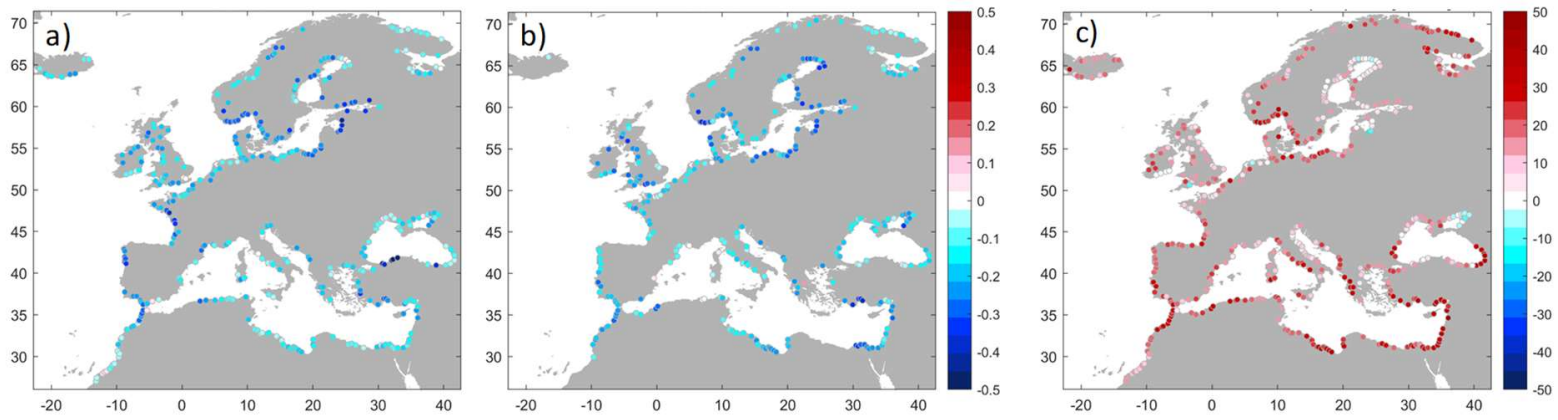

Figure S4. Differences in the Kendall's correlation coefficients $(p<0.05)$ between $S-W$ compound extreme events using daily data and hourly data. Conditional extreme events are selected using POT approach (3 events per year), with either (a) $\mathrm{S}$ or (b) W as the dominant variable. (c) indicates the differences in the number of co-occurring events when using daily versus hourly S-W data. 
a)

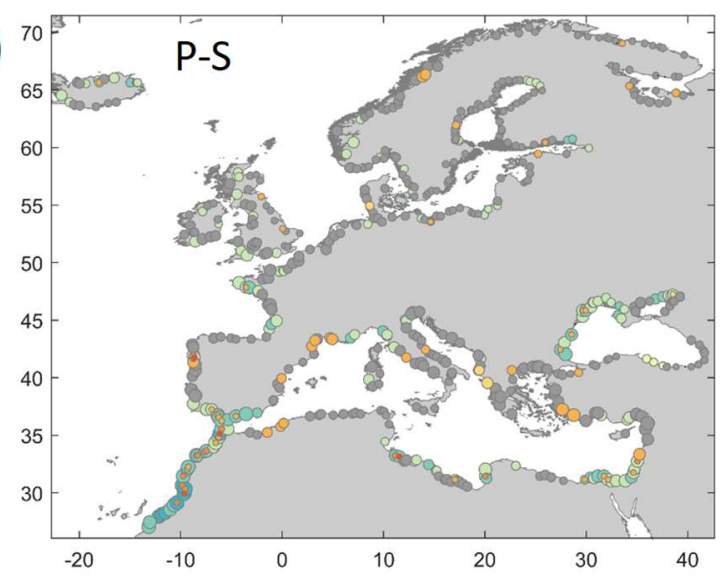

c)

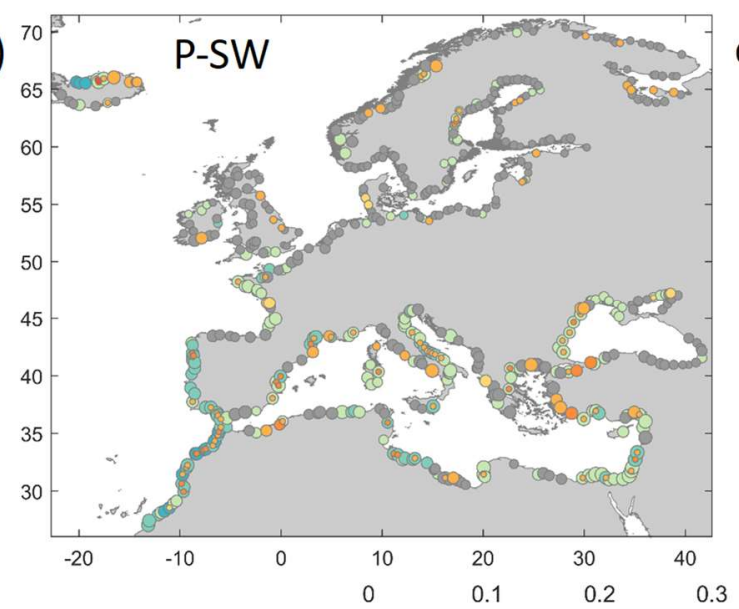

b)

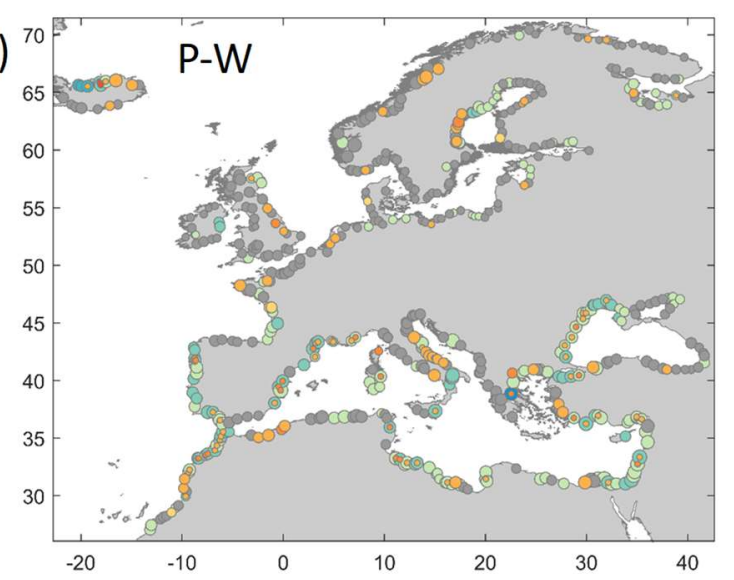

d)

Figure S5: Kendall's correlation coefficients $(\mathbf{p}<0.05)$ between multivariate extremes selected using the POT3 approach and the join occurrences between (a) P-S, (b) P-W, (c) P-SW and (d) S-W. Correlations between compound events are selected when variable 1 is the dominant driver is represented on a red scale while the blue scale denotes the correlations obtained when variable $\mathbf{2}$ is the dominant driver. The size of the circles in blue scale represents the joint occurrences (maximum=100). If the correlation is insignificant when variable 2 is the conditioning variable, the size of the circle on red scale represents the joint occurrences. When both correlation coefficients are insignificant, the size of the grey circle represents the joint occurrences. 
a)

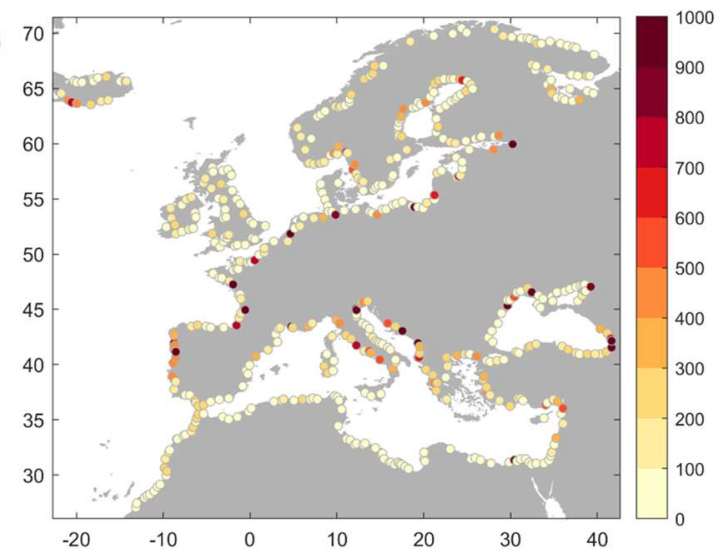

c)

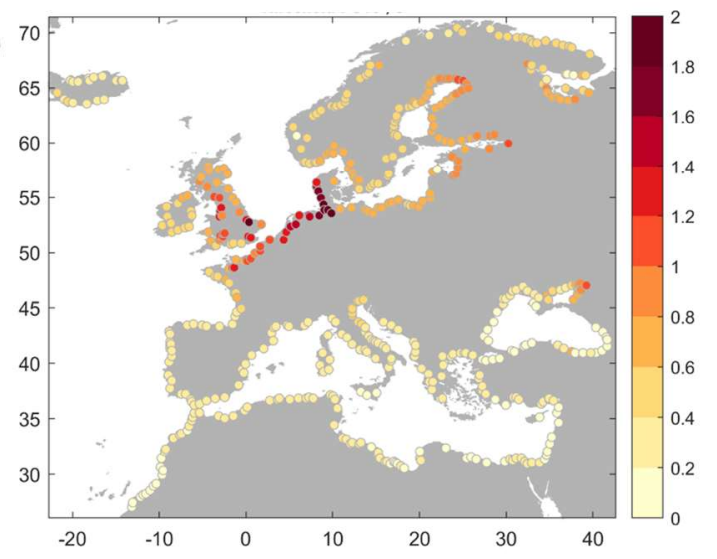

b)

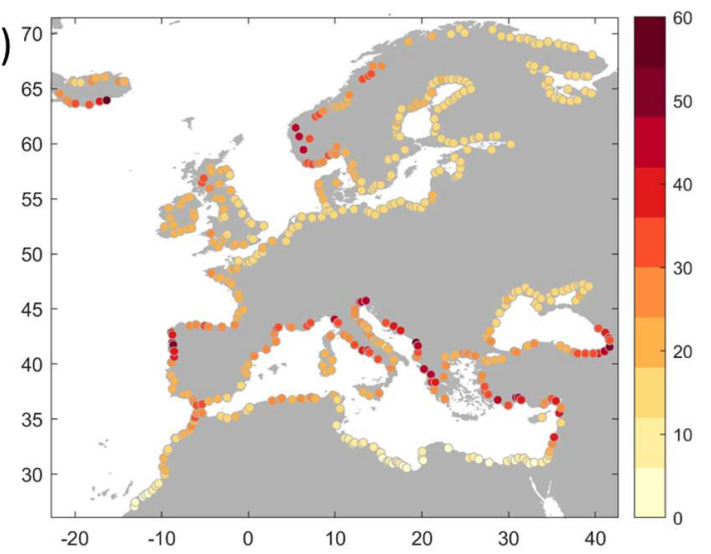

d) 70

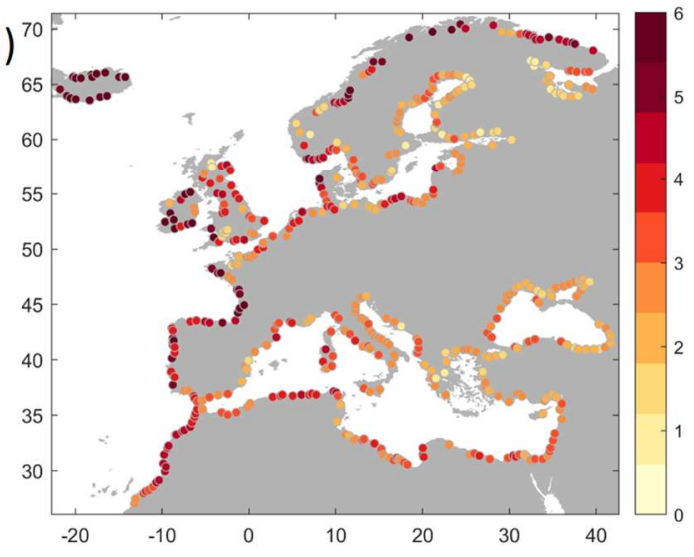

Figure S6. Threshold that selects 3 events/year using POT for (a) $Q$, (b) $P$, (c) $S$ and (d) W, in m3/s, mm, m and m, respectively. 

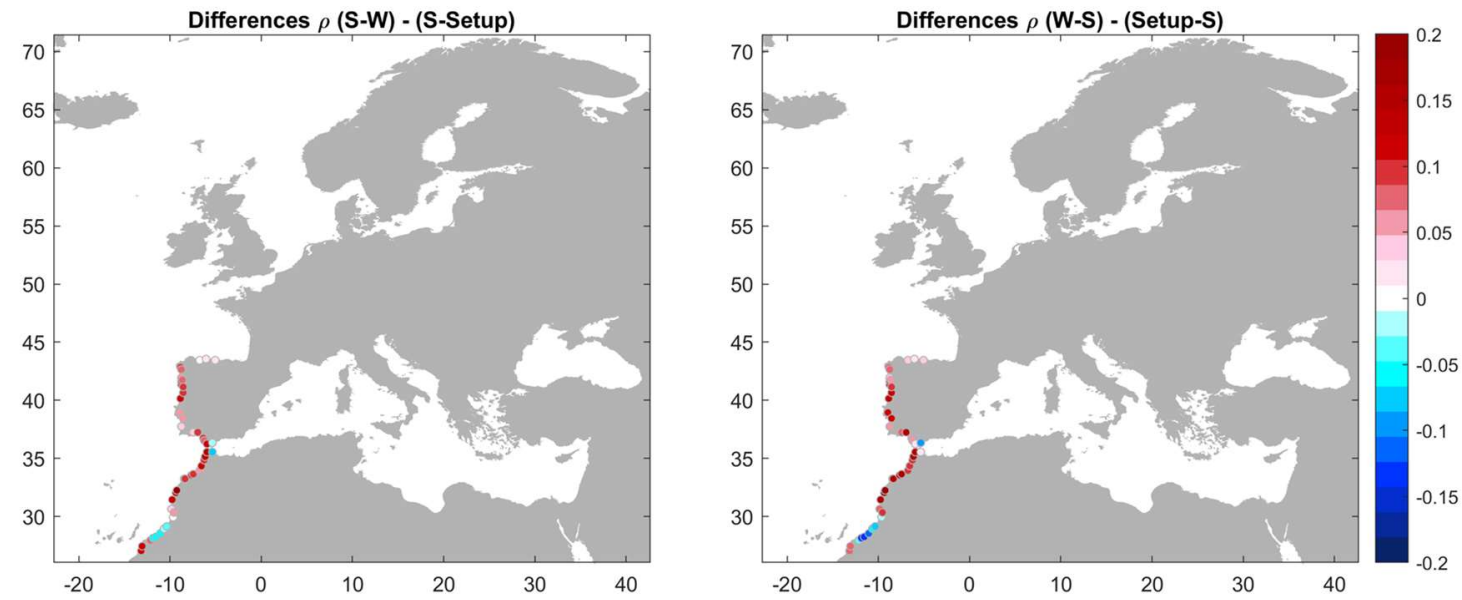

Figure S7. Differences in the Kendall's correlation coefficient between the pairs of variables S-W and S-Setup, when either S (left panel) or W/Setup (right panel) are used as the dominant variable in the conditional sampling. 